\title{
REDE DE ATENÇÃO EM SAÚDE MENTAL NA PERSPECTIVA DOS COORDENADORES DE SERVIÇOS DE SAÚDE
}

\author{
MENTAL HEALTH CARE NETWORK FROM THE VIEW OF HEALTH SERVICE COORDINATORS
}

\author{
Lucilene Gama Paes ${ }^{1}$ \\ Maria Denise Schimith ${ }^{2}$ \\ Tatiane Muniz Barbosa ${ }^{3}$ \\ Liane Beatriz Righi ${ }^{4}$
}

Resumo Trata-se de um estudo descritivo exploratório com abordagem qualitativa, que teve como objetivo compreender como coordenadores de serviços de saúde, envolvidos no cuidado dos sujeitos em sofrimento psíquico, percebem a organização da rede de atenção em saúde mental de Santa Maria, Rio Grande do Sul, Brasil. Os dados foram coletados por meio de entrevista semiestruturada e de vinhetas nos meses de abril e maio de 2008. Participaram da pesquisa dez coordenadores de serviços de saúde. Os dados foram trabalhados mediante análise temática e, posteriormente, agrupados em três núcleos de sentido: o entendimento dos fluxos assistenciais; o funcionamento da rede; e as dificuldades e potencialidades vislumbradas. Evidenciou-se uma rede em processo de construção, destacando-se a existência de movimentos que visam à melhoria da articulação entre os serviços e consequente qualificação da assistência para os usuários da rede de cuidado em saúde mental. Propõe-se um estreitamento das relações entre os serviços, intensificando a articulação entre a atenção primária, os serviços substitutivos e a atenção hospitalar. Destaca-se o papel da gestão nesse processo, considerando a necessidade de envolvimento de diferentes setores para a efetivação de um serviço de atenção à saúde mental mais qualificado.

Palavras-chave atenção à saúde; saúde mental; acesso aos serviços de saúde.
Abstract This is an exploratory descriptive study based on a qualitative approach which purpose was to understand how health service coordinators involved in providing care to individuals in psychological distress perceive the organization of the mental health care network in Santa Maria, state of Rio Grande do Sul, southern Brazil. Data were collected through semi-structured interviews and vignettes in the months of April and May 2008. Ten health service coordinators participated in the study. The data were processed through thematic analyses and, subsequently, grouped into three clusters of meaning: The understanding of care flows, network operation, and of the difficulties and potential envisioned. The results showed a network under construction, highlighting the existence of movements aimed at improving coordination between the services and the resulting quality of care provided to the users of the mental health care network. The authors propose a reinforcement of the relations between the services, heightening the relationship between primary care, substitutive services, and hospital care. Emphasis is put on the role played by management in the process, considering the need for the involvement of different sectors in order to attain a more qualified mental health care service.

Keywords health care; mental health; access to health services. 


\section{Introdução}

A Constituição Federal promulgada em 1988 trouxe grandes avanços na atenção à saúde no Brasil, com a criação do Sistema Único de Saúde (SUS). Naquele momento, a saúde deixou de ser direito apenas de uma parcela da população para tornar-se um direito de todos e dever do Estado.

Contudo, para que esse direito se efetive, as ações e os serviços públicos de saúde devem desenvolver ações de promoção, proteção e recuperação da saúde. Também é importante que integrem uma rede regionalizada e hierarquizada, sustentada por diretrizes organizativas e princípios doutrinários que assentam o SUS (Vasconcelos e Pasche, 2006).

Paralelamente à Reforma Sanitária, desenvolveu-se a Reforma Psiquiátrica, um movimento histórico de caráter político, social e econômico que teve início na década de 1970 (Gonçalves e Sena, 2001). Com o pressuposto ético da cidadania do sujeito em sofrimento psíquico e de sua inserção na comunidade e na família, a Reforma Psiquiátrica propôs mudanças no modelo assistencial, sendo uma das pautas centrais a substituição progressiva dos manicômios por outras práticas terapêuticas.

O conceito de desinstitucionalização defendido pela Reforma Psiquiátrica não se restringe à substituição do hospital e à criação de serviços substitutivos. Vai além, exigindo um deslocamento das práticas de cuidado em saúde mental para a comunidade (Gonçalves e Sena, 2001), com a construção e implementação de novas formas de cuidado. O sujeito passa então a ser visto em sua totalidade, requerendo demandas de trabalho, de lazer, de cuidados, de relações e afetos (Lancetti e Amarante, 2006).

A saúde mental foi pioneira na produção de novas formas de cuidado, das quais se destacam os Projetos Terapêuticos (PTs), que procuram singularizar o indivíduo ou o coletivo, com um conjunto de propostas terapêuticas articuladas com uma equipe multiprofissional e participação do sujeito (Brasil, 2007).

O conceito de Rede de Atenção à Saúde (Brasil, 2011) é compreendido como o conjunto de ações e serviços de saúde articulados em níveis de complexidade crescente, com a finalidade de garantir a integralidade da assistência à saúde. A partir desse conceito pode-se reconhecer que as práticas de cuidado em Saúde Mental requerem a mesma lógica de organização.

Este estudo justifica-se pela necessidade de revisar as ações de cuidado, visando a práticas mais articuladas e centradas nas reais necessidades dos usuários. Destaca-se a concepção de linhas de cuidado, cuja proposta fundamenta-se na organização da gestão e de práticas assistenciais centradas na inclusão de pessoas em uma rede de práticas cuidadoras em saúde (Ceccim e Ferla, 2006). 
Assim, o objetivo do estudo foi compreender como coordenadores de serviços de saúde envolvidos no cuidado dos sujeitos em sofrimento psíquico percebem a organização da rede de atenção em saúde mental.

\section{Percurso metodológico}

O estudo caracteriza-se como uma pesquisa descritiva, exploratória com abordagem qualitativa, realizado no município de Santa Maria, interior do estado do Rio Grande do Sul.

Santa Maria situa-se no centro do estado e possui uma população de 261.031 habitantes (IBGE, 2010), a qual está distribuída entre 21 regiões sanitárias, sendo 13 na zona urbana e oito na rural. A rede de atenção à saúde do município conta com 14 unidades de saúde da família (USFs), 17 unidades básicas de saúde (UBSs), uma unidade móvel, 12 serviços especializados de apoio e três pronto-atendimentos municipal (PA), sendo um adulto, um infantil e um odontológico.

Entre os serviços especializados, estão os substitutivos, como o Centro de Atenção Psicossocial para Álcool e Drogas (CAPS ad); Centro de Atenção Psicossocial para Sofrimento Mental Grave (CAPS II), Centro de Atenção Psicossocial para Infância e Adolescência (CAPSi) e Ambulatório Municipal de Saúde Mental. Sedia ainda o Hospital Universitário de Santa Maria (HUSM), referência para atendimento de emergência, internação e serviço ambulatorial.

A escolha dos serviços para coleta de dados seguiu a lógica da rede de atenção à saúde, identificando-se serviços de diferentes densidades tecnológicas: atenção primária, serviços substitutivos, atenção hospitalar e gestão. $\mathrm{Na}$ atenção primária, selecionaram-se UBSs de diferentes distritos sanitário do município. Entre as USFs, optou-se por aquelas que apresentassem mais de uma equipe de saúde da família na mesma estrutura física. $\mathrm{O}$ ambulatório do HUSM e o CAPS II foram escolhidos por serem referência no atendimento em saúde mental na atenção hospitalar e em serviços substitutivos respectivamente. Entrevistaram-se ainda profissionais da gestão municipal e regional.

Dessa forma, incluíram-se neste estudo dez serviços de saúde: três USFs, três UBSs, um CAPS II, um ambulatório de Psiquiatria e duas instâncias de gestão, uma municipal e outra regional.

Os profissionais entrevistados exerciam o cargo de coordenadores dos serviços e foram abordados nos meses de abril e maio de 2008, por meio da entrevista semiestruturada e de vinhetas. As vinhetas traziam uma breve descrição de uma situação hipotética, às quais os respondentes eram solicitados a apresentar suas condutas, dificuldades enfrentadas e a relação com os demais serviços da rede de atenção em saúde mental. Essas descrições 
foram estruturadas e adaptadas à realidade de cada local e objetivavam extrair informações sobre as percepções, as opiniões ou o conhecimento dos respondentes sobre o fenômeno (Polit, Beck e Hungler, 2004). A entrevista semiestruturada, utilizada de forma concomitante às vinhetas, era composta por cinco questões que versavam sobre o entendimento, funcionamento, dificuldades e facilidades do sistema de referência e contrarreferência em saúde mental do município.

A coleta realizou-se após esclarecimento dos objetivos e métodos da pesquisa, formalizado por meio da assinatura do termo de consentimento livre e esclarecido. As entrevistas foram gravadas e posteriormente transcritas, codificadas e enumeradas de Ent-1 a Ent-10, para o anonimato dos entrevistados.

Os dados foram trabalhados por meio de uma análise temática. Essa proposta de interpretação leva-nos a descobrir os 'núcleos de sentido' presentes na comunicação, que representam o objeto investigado (Minayo, 2007, p. 316). Para auxiliar nessa interpretação, seguiram-se os passos de pré-análise; exploração do material; tratamento dos resultados obtidos; e interpretação.

Os dados foram categorizados e posteriormente reagrupados em três núcleos de sentido, tendo por base o referencial teórico deste estudo: o entendimento dos fluxos assistenciais, o funcionamento da rede e as dificuldades e potencialidades vislumbradas.

A condução ética foi garantida durante todo o processo desta pesquisa, seguindo as orientações da resolução n. 196/96, do Conselho Nacional de Saúde (Brasil, 1996). Obteve-se a aprovação das instâncias envolvidas na pesquisa, como o Núcleo de Educação Permanente da Saúde da Secretaria Municipal de Saúde, a Direção de Ensino Pesquisa e Extensão do HUSM e a 4a Coordenadoria Regional de Saúde. O projeto foi aprovado pelo Comitê de Ética da Universidade Federal de Santa Maria.

\section{O entendimento dos fluxos assistenciais}

Evidenciou-se um entendimento dos fluxos assistenciais pautado na lógica do encaminhamento: usuários que não conseguem ter suas necessidades atendidas pela atenção primária são encaminhados para outros serviços do município.

O que não é resolvido dentro das unidades tem que ter para onde mandar os pacientes, a referência (...) muitas vezes ele sai com aquele encaminhamento no bolso, a gente não sabe se ele foi. Muitas vezes ele volta uns meses depois, e aí tu ficas sabendo que ele não fez nada com aquele encaminhamento (Ent-7). 
Percebe-se que a continuidade da resolução do caso é uma preocupação do trabalhador. No entanto, esse sistema de repasse dos usuários entre as unidades da rede assistencial, com enfoque no encaminhamento, reforça a prática de uma assistência fragmentada, com pouca articulação entre os serviços e faz com que o usuário fique perdido no sistema. As formas de encaminhamento praticadas no município contribuem pouco para o princípio da integralidade e para o avanço da Reforma Psiquiátrica, pois o processo de implantação da rede de atenção em saúde mental sob a lógica dos encaminhamentos pode desencadear a destituição de vínculo e a desresponsabilização (Zambenedetti e Perrone, 2008).

Outro aspecto relevante desvelado nas entrevistas foi a existência de distinção entre o 'encaminhar' e o 'referenciar'. O primeiro apresenta-se como o ato propriamente dito de 'passar' o usuário a outro serviço; o segundo, por sua vez, relaciona-se com a ideia de transmissão de informações.

(...) então, hoje em dia, é tudo a mesma ficha de referência e contrarreferência e do encaminhamento. A gente está separando, (...) iria junto o encaminhamento, com usuário, e a referência e a contrarreferência, justamente para reforçar essa coisa de que a referência é para tirar os dados, para tu poderes informar sobre esse paciente. Não é só botar o nome e o endereço, isso é o encaminhamento (Ent-8).

Os profissionais apontam o sistema de referência e contrarreferência como de fundamental importância para a qualidade da assistência, pois permite ao usuário um acompanhamento mais resolutivo e uma continuidade no seu atendimento.

(...) eu vejo a referência e a contrarreferência como uma continuidade. Não é só ficar 'vai para cá e vai para lá', entende? Que seja realmente a referência, mas que a pessoa possa retornar para o outro local para continuar sendo acompanhada (Ent-1).

A referência e contrarreferência são fundamentais para o alcance da resolutividade que os profissionais devem procurar alcançar dentro do SUS (Ent-9).

Verificou-se a necessidade de articulação entre os diversos coordenadores de serviços nos diferentes serviços de saúde, pois teria grande impacto para diminuir a fragmentação do cuidado. Isso leva a crer na importância de se elaborar instrumentos e estratégias que garantam fluxos diversos entre os níveis de atenção, considerando as USFs e UBSs locais onde vínculo e o acompanhamento dos usuários devem ser permanentes (Franco e Magalhães Jr., 2006). 


\section{O funcionamento da rede}

No município em questão, a rede de atenção em saúde mental encontrava-se num momento de estruturação, de elaboração de conceitos e de construção de novas práticas. Este fato se confirma com a fala dos profissionais ao mencionarem este processo de construção e a necessidade de se produzirem ações capazes de proporcionar articulação.

Ter uma rede totalmente construída, não tem, mas eu posso dizer, assim, que está em fase de construção (...). Eu acho que devemos começar a discutir intersetorialmente, estamos tentando fazer esse exercício (...) discutir com a assistência, com a justiça, com a segurança, com a educação, com diversas áreas, que a gente possa também começar a rever o seu papel, a importância do trabalho em conjunto (Ent-1).

Os entrevistados relataram que a relação entre os serviços baseia-se na utilização de um instrumento formal - um formulário - e na ligação telefônica, utilizados para auxiliar e assessorar os serviços.

(...) ele [o sistema de referência e contrarreferência] funciona a partir do encaminhamento. Encaminhamento formal (...) ele funciona a partir da ligação telefônica, de tu discutires o caso, apresentar o caso, ele funciona muito em termos de assessoria (Ent-6).

O contato com as outras instituições se dá por escrito, via paciente. Às vezes vem em anexo algum outro papel que traz informações ou contato telefônico, que usamos muito (Ent-2).

O formulário é considerado de pouca eficácia, pois não se apresenta organizado de maneira a permitir que informações importantes sejam repassadas entre as equipes.

O normal, quer dizer, a fichinha de referência e contrarreferência, tu preenches os dados do paciente, tem quatro linhas, preenches o histórico, o diagnóstico, quando chega numa unidade, ao ver aquilo, não vai se ter muita noção do que vem (Ent-8).

Dessa forma, as informações e os usuários perdem-se dentro do sistema, resultando na ausência de resolutividade. Percebe-se que os usuários têm buscado os serviços a partir de sua condição de acessibilidade, rapidez e capacidade de resolução de problemas, construindo fluxos assistenciais com diversas portas de entrada. O hospital apresentou-se como uma porta de entrada para as urgências, mas trata-se de uma porta em que o usuário entra, recebe alguma resposta e sai. Não se trata de uma porta que garanta a entrada para a continuidade da assistência. 
(...) se precisa de uma consulta psiquiátrica hoje, tu vais ao hospital, no plantão. Agora, na rede, vai demorar bastante (Ent-6).

O hospital é utilizado apenas em situações de emergência, às vezes o usuário nem chega até a unidade, a comunidade já encaminha para o hospital (Ent-2).

Verifica-se que esses profissionais identificam a rede de atenção primária como o lugar que deveria garantir a acessibilidade, porém reconhecem que o hospital ainda ocupa esse espaço quando a necessidade é de especialista. Desta forma para acessar especialistas na saúde mental, a população do município estudado procura-os no hospital, mostrando que a via estruturada do fluxo assistencial ainda não atende as necessidades do sujeito em sofrimento psíquico.

Ao acessar o sistema por uma das diferentes portas de entrada, identificadas por meio das entrevistas, o usuário inicia o seu caminhar na rede de atenção do município.

Desse modo, considerando os relatos dos entrevistados, foi possível constatar que, no município, existem quatro formas de acesso aos serviços: atenção primária (UBSs e USFs), serviços de atenção especializada (CAPSs), ambulatório municipal de saúde mental e atenção hospitalar. No entanto, isso não tem garantido a continuidade da assistência, pois o usuário realiza o seu itinerário de forma solitária, sem o acompanhamento efetivo de nenhum serviço.

Conforme já mencionado, a atenção hospitalar permanece como uma importante porta de entrada para as situações de urgência, mas sua relação com os serviços de atenção primária é pequena ou quase inexistente. Contudo, percebe-se uma tentativa de aproximação com os serviços especializados (CAPS e ambulatório) efetivada por meio dos encaminhamentos realizados após o tratamento hospitalar.

De acordo com os entrevistados, os serviços substitutivos (CAPS e ambulatório municipal) encontram-se 'estrangulados' pela grande demanda e, assim, não conseguem dar conta de todos os atendimentos a eles encaminhados. Dessa forma, as evidências ratificam que a assistência à saúde, nas especialidades de média e alta complexidade e apoio diagnóstico, são em geral, locais de estrangulamento do sistema de saúde (Franco e Magalhães Jr., 2006).

Com isso, os usuários são avaliados quanto à possibilidade de espera pelo atendimento, incluindo-se no que os profissionais chamam de 'demanda reprimida'.

Às vezes, devido à falta de vaga, quando se encaminha para o ambulatório municipal, o paciente fica na lista de espera aguardando. Que é nosso grande problema (Ent-5).

Devido à demora no atendimento com o psiquiatra, o paciente é avaliado quanto à possibilidade de aguardar ou não, processo realizado pela triagem (Ent-2). 
Os poucos CAPSs que existem absorvem alguns poucos pacientes. Tem demanda reprimida, vamos dizer assim (Ent-4).

Aqueles que permanecem nas filas, 'a demanda reprimida', retornam aos serviços de atenção primária, gerando uma nova demanda. Eles se apresentam, segundo os entrevistados, como usuários 'poliqueixosos'. Essa é uma dificuldade enfrentada pelos profissionais, mas principalmente pelos usuários, que, não tendo sua necessidade atendida, 'vagam' pelo sistema, resultando num inchaço dos serviços decorrente da ausência de resolutividade e potencialização do sofrimento dos usuários e familiares.

(...) as pessoas usam muito do nosso serviço, muitas vezes sem necessidade, (...) as pessoas vão para o pronto socorro, sem necessidade nenhuma (...) então o pronto socorro está superlotado, sem necessidade. Então isso acontece aqui também, as pessoas vêm procurar, vêm consultar três vezes por semana, o que é desnecessário, muitas vezes (Ent-7).

Identifica-se, nessa fala, que alguns profissionais sentem-se incomodados com o excesso de consultas de alguns usuários, mas os motivos que os levam a procurar o serviço tantas vezes, numa mesma semana, não são questionados. Situações como essas podem desvelar a complexidade dos sujeitos que utilizam os serviços de saúde, além de demonstrar os limites da prática clínica centrada na doença (Brasil, 2007).

Situação similar pode ser evidenciada em outro estudo, que teve como objetivo analisar o trabalho de uma equipe de saúde da família, mostrando que alguns coordenadores de serviços caracterizam esses usuários como dependentes emocionais. Talvez as necessidades de saúde desses usuários não estejam sendo ouvidas e atendidas, o que acarreta o retorno insistente ao serviço (Schimith e Lima, 2004).

No CAPS, a entrada do usuário, segundo os entrevistados, acontece pelo acolhimento, seja daqueles que chegam espontaneamente no serviço, seja dos encaminhados pelo serviço hospitalar e pela atenção primária. Do CAPS, os usuários que não possuem transtorno mental grave, ou que já estão estabilizados, são encaminhados para o ambulatório municipal ou USF/UBS.

Nesse contexto, a equipe de saúde da família deveria somar-se ao projeto terapêutico, pelas características de trabalho esperadas, permitindo a realização do acolhimento e o desenvolvimento de vínculo entre os familiares, sujeitos em sofrimento psíquico e equipe de saúde. A atenção primária tem um importante papel no processo de reinserção social, pois está imersa nos territórios, vivenciando a realidade dos sujeitos e de suas famílias (Figueiredo e Campos, 2008). 
No entanto, de acordo com os dados da pesquisa, nota-se que a atenção primária aparece como uma porta de entrada muito incipiente. O usuário que procura esses serviços é geralmente encaminhado para atendimento em outros pontos da rede (CAPS e ambulatório), como se a atenção primária não pudesse responsabilizar-se por transtornos relativos à saúde mental, desconsiderando o sujeito que existe por trás da doença.

Percebe-se a necessidade de transformação das práticas de cuidado à saúde dos sujeitos em sofrimento psíquico. Defende-se que elas sejam compreendidas e trabalhadas em sua integralidade, focalizadas na promoção da qualidade de vida. Para isso, os serviços de saúde também precisam transformar-se, a fim de promover a integração e interação dos profissionais nos diferentes nós da rede de atenção à saúde mental.

\section{As dificuldades e potencialidades vislumbradas}

No que tange aos aspectos positivos e negativos na rede de atenção do município, é quase unânime a opinião de que ainda há muito a ser construído. As relações entre as equipes precisam ser trabalhadas e estas precisam ser mais bem instrumentalizadas.

(...) existem poucos lugares que realmente absorvem o paciente quando a gente reencaminha (Ent-4).

O enfermeiro e os clínicos deveriam ser capacitados, porque às vezes é só um descontentamento, uma orientação para a realização de outras atividades, e acabam sendo realizados encaminhamentos desnecessários (Ent-2).

Pode-se verificar que existe uma relação estreita entre as principais dificuldades relatadas pelos profissionais, ou seja, o surgimento de uma predispõe o aparecimento de outra. Segundo eles, o déficit de profissionais especializados é o ponto crítico dessa rede, o que gera falta de vagas para os usuários nos serviços de saúde e, como consequência, a lista de espera, a qual implica demora no atendimento. E isso se torna um dos principais problemas para a prática da integralidade.

(...) um número alto assim [referindo-se à grande demanda] para um número reduzido de profissionais que se tem nessa área de saúde mental. (...) os pacientes demoram muito para ser agendados (Ent-7).

(...) o acesso à consulta médica é que é muito restrito, tornando-se uma dificuldade. É uma questão quantitativa (Ent-6). 
(...) a demora no atendimento do psiquiatra (...) Esse paciente que aguarda não sai de dentro do posto com várias queixas, como dor de estômago (Ent-2).

Outra dificuldade referida relaciona-se com a compreensão dos profissionais sobre o funcionamento do sistema de saúde, ora pela formação deficiente, ora pela resistência a mudanças apresentada pelos coordenadores de serviços.

(...) dificuldade técnica que vem da compreensão dos coordenadores de serviços de como fazer isso, da importância disso. Temos uma formação que não trabalha essas questões, uma formação mais voltada ao procedimento, e não tanto para essa coisa de serviço, para essa relação que tem que ter (Ent-8).

(...) uma das grandes dificuldades é até o próprio trabalhador de saúde muitas vezes, a própria resistência (...) essa grande resistência, essa grande dificuldade às vezes é por falta de preparação, é por falta de uma própria formação do trabalhador, sabe, de estar preparado para isso (Ent-1).

A educação permanente em serviço deveria ser estimulada no SUS, pois a formação dos profissionais de saúde configura-se como um desafio, já que a maioria dos coordenadores de serviços continua sendo formada distante do debate e da construção de políticas de saúde (Brasil, 2004).

Aproximar a aprendizagem dos espaços de trabalho é uma necessidade sentida pelos próprios profissionais no dia a dia. Eles relatam que o desconhecimento dos demais serviços que o município dispõe e a falta de articulação entre eles emergem como uma dificuldade.

(...) tem a velha dificuldade de dois grupos [referindo-se à Secretaria Municipal de Saúde e à Atenção Hospitalar] trabalhando com objetivos comuns, o entendimento entre os dois grupos também é encrencado, não flui. Até por falta de conhecimento um do outro (Ent-4).

Tem a questão também de não saber quais são as referências, não saber para onde vai, quem vai, o que vai, quem que pode ajudar, o que pode e o que não pode, tem isso ainda (Ent-8).

Apesar das dificuldades na formação, os discursos revelam o surgimento de novos profissionais como aspecto positivo na construção da rede de cuidados em saúde mental, haja vista a possibilidade de inserção de novas interrogações, que podem despertar pensamentos e resultar em novas intervenções. 
(...) há facilidades também na entrada de novos profissionais no serviço (...) há mudança no serviço quando começa a entrar profissionais novos (...) o serviço passa por um novo olhar, eu acho que isso facilita (Ent-1).

Vislumbram-se, portanto, dificuldades de ordem estrutural, mas principalmente de natureza organizacional dos serviços e de quantitativo de coordenadores de serviços. Além disso, a relação existente entre a implementação da rede e a formação dos profissionais faz com que as instituições de saúde e as instituições de ensino superior também sejam responsabilizadas pela construção do SUS, mesmo que isso apareça indiretamente na fala dos profissionais.

O município tem procurado implementar alternativas para tornar realidade a troca de informações, a articulação entre os serviços e profissionais, além de qualificar os profissionais. Entre as alternativas os profissionais destacam os encontros de "saúde mental na roda" e as "equipes de apoio matricial.

(...) eu acho que (...) um processo de saúde mental na roda,que começou a pensar, a idealizar a rede, (...) também (...) com um processo agora de equipe de matriciamento, acho que isso é uma coisa interessante (Ent-1).

Até março de 2008, a equipe (...) estava participando da saúde mental na roda, que é uma capacitação para a equipe. Eram realizados encontros mensais com a comissão, para trazer o que é saúde mental e como os serviços deveriam funcionar. Também foram feitas oficinas (Ent-2).

Os encontros de "saúde mental na roda", propiciados pela Comissão de Saúde Mental do município, têm proporcionado momentos de discussão entre os profissionais de instituições de saúde distintas. Os objetivos são conhecer a rede, permitir uma visibilidade entre os serviços e as pessoas que os compõem, discutir a rede de saúde mental e procurar estratégias para lidar com a realidade apresentada em relação à saúde no município (Zambenedetti e Perrone, 2008).

A equipe de apoio matricial desponta como uma alternativa para alguns dos problemas vivenciados na implementação da rede no município, apontado como um 'projeto audacioso' pelo discurso dos profissionais.

Até um projeto mais audacioso, eu diria, seriam as equipes de apoio matriciais, que eu sei que já há um movimento nesse sentido. Seria excelente, porque, daí, seria por regiões da cidade que estariam cobertas pela equipe, seria um serviço com uma referência regional, seria muito bom, seria mais fácil para tu atenderes a demanda (Ent-6). 
O apoio matricial pretende assegurar retaguarda especializada a equipes e profissionais encarregados da atenção dos problemas de saúde da população, emergindo como um trabalho complementar aos sistemas hierarquizados de referência e contrarreferência (Campos e Domitti, 2007).

Contudo, verifica-se que a atenção em saúde mental requer dos profissionais a capacidade de trabalho em equipe, certa predisposição subjetiva para receber e fazer críticas e para a tomada de decisões de modo compartilhado (Campos e Domitti, 2007). Da mesma forma, reconhecer a interdependência dos atores e das organizações (Hartz e Contadriopoulos, 2004), permitindo que a assistência aos sujeitos em sofrimento psíquico seja integral e resolutiva.

\section{Palavras finais}

Para estudar a rede de atenção em saúde mental do município e os seus fluxos assistenciais, o sistema de referência e contrarreferência foi utilizado como analisador da conectividade entre os serviços. Esse sistema vem recebendo críticas com relação à sua praticidade e eficácia na organização do fluxo de usuários dentro da rede de atenção em saúde, ratificadas por esta pesquisa.

A integralidade da assistência só pode ser garantida pela forma como as conexões são estabelecidas e as alternativas utilizadas para sua melhor implementação. O que de fato está preconizado é uma troca de informações, é um contato entre profissionais com vistas a qualificar o cuidado ao usuário, seja ele realizado por um formulário, seja pelo contato telefônico ou pelo contato direto entre os profissionais e equipes de matriciamento.

Evidenciou-se uma rede em processo de construção, com a existência de movimentos que visam à melhoria da articulação entre os serviços e à consequente qualificação da assistência a esse público, por meio da implementação dos encontros de 'saúde mental na roda'.

Entretanto, grandes desafios ainda precisam ser superados. As equipes precisam ser mais bem instrumentalizadas, de modo a qualificá-las para o trabalho com o sujeito em sofrimento psíquico na comunidade; incentivá-las à criação de novas formas de cuidado que não sejam pautadas apenas na prática curativa, desconstruindo e construindo novos paradigmas de cuidado; desvencilhá-las de concepções pré-formuladas e apresentá-las aos diferentes serviços disponibilizados no município, destacando a importante posição que as instituições formadoras assumem nesse processo.

Os serviços substitutivos encontram-se lotados e com demanda reprimida, apesar de serem serviços relativamente novos no município. Propõe-se um estreitamento das relações entre os serviços, intensificando sua articulação 
com a atenção primária, a atenção hospitalar e os serviços substitutivos, como alternativa ao estrangulamento em que esses serviços se encontram, além de permitir continuidade da assistência ao usuário.

Contudo, vale destacar o importante papel que a gestão deve assumir nesse processo, considerando a necessidade de envolvimento de diferentes setores para a efetivação de um serviço de atenção à saúde mental humanizado, resolutivo e pautado na promoção de saúde e qualidade de vida de usuários e familiares.

É importante verificar os tipos de sistema empregatício utilizados. A rotatividade de pessoal acarreta dificuldade em estabelecer vínculo, considerando que o vínculo estabelecido entre usuário e equipe de saúde foi apontado como um ponto positivo na construção da rede.

Torna-se necessário repensar a rede de atenção em saúde mental no município estudado, vislumbrando-a com diferentes portas de entrada, a fim de focalizar a atenção nas pessoas e em suas necessidades, uma vez que o usuário vai acessar os diversos serviços de maneira constante, precisando, assim, caminhar nessa rede.

Ressalta-se que este estudo apresentou a percepção de uma parte dos fluxos assistenciais existentes neste município, focando-se à normativa dos serviços, o que caracteriza os limites deste estudo. Embora tenha colaborado no entendimento nesse sentido da rede, são necessárias novas investigações para buscar compreender como se dão as conexões entre os sujeitos coordenadores de serviços na implementação de redes, como estratégia na realização do cuidado integral.

\section{Colaboradores}

Lucilene Gama Paes participou da concepção, coleta de dados, análise de dados e redação do artigo. Maria Denise Schimith atuou na concepção, orientação, redação do artigo e revisão. Tatiane Muniz Barbosa e Liane Beatriz Righi colaboraram na revisão. 


\section{Notas}

${ }^{1}$ Enfermeira na Secretaria Municipal de Saúde de Florianópolis, Santa Catarina. Brasil. Mestranda do Programa de Pós-graduação em Enfermagem da Universidade Federal de Santa Maria (UFSM). <lucilenepaes@yahoo.com.br>

Correspondência: Rua José João Martendal, 315, apto 108, Trindade, CEP 88040-420, Florianópolis, Santa Catarina, Brasil.

2 Professora Assistente do Departamento de Enfermagem do Centro de Ciências da Saúde da Universidade Federal de Santa Maria (UFSM), Santa Maria, Rio Grande do Sul, Brasil. Doutoranda em Ciências da Saúde pelo Dinter Novas Fronteiras da Universidade Federal de São Paulo (Unifesp/UFSM). <ma.denise2011@gmail.com>

3 Professora dos cursos de Psicologia e Odontologia da Universidade do Planalto Catarinense (Uniplac), Lages, Santa Catarina, Brasil. Mestre em Saúde e Gestão do Trabalho pela Universidade do Vale do Itajaí (Univali). < tatibitatil1@yahoo.com.br>

4 Professora do Departamento de Ciências da Saúde, do Centro de Ensino Superior Norte, da Universidade Federal de Santa Maria (Cesnor/UFSM), Palmeira das Missões, Santa Catarina, Brasil. Doutora em Saúde Coletiva pela Universidade Estadual de Campinas (Unicamp).<lianerighi@gmail.com>

\section{Referências}

BRASIL. Conselho Nacional de Saúde. Resolução n. 196, de 10 de outubro de 1996. Dispõe sobre as diretrizes e as normas regulamentadoras de pesquisa envolvendo seres humanos. Brasília: CNS, 1996.

Ministério da Saúde. Secretaria Executiva. Núcleo Técnico da Política Nacional de Humanização. HumanizaSUS: documento base para gestores e coordenadores de serviços no SUS. Brasília: Ministério da Saúde, 2004. Disponível em: $<$ http://bvsms.saude.gov.br/bvs/publicacoes/ humanizaSus_doc_base.pdf $>$. Acesso em: 2 maio 2009.

. Ministério da Saúde. Secretaria de Atenção à Saúde. Núcleo Técnico da Política Nacional de Humanização. Clínica ampliada, equipe de referência e projeto terapêutico singular. 2. ed. Brasília: Ministério da Saúde,
2007. Disponível em: <www.ufjf.br/hu/files/ 2009/10/projetos_terapeuticos.pdf > . Acesso em: 20 maio 2008.

Presidência da República. Casa Civil. Subchefia para Assuntos Jurídicos. Decreto n. 7.508, de 28 de junho de 2011. Regulamenta a Lei n. 8.080, de 19 de setembro de 1990, para dispor sobre a organização do Sistema Único de Saúde - SUS, o planejamento da saúde, a assistência à saúde e a articulação interfederativa. Disponível em: <www.planalto.gov.br/ccivil_03/ _ato2011-2014/2011/decreto/D7508.htm>. Acesso em: 15 set. 2012.

CAMPOS, Gastão W. S.; DOMITTI, Ana C. Apoio Matricial e Equipe de Referência: uma metodologia para gestão do trabalho interdisciplinar em saúde. Cadernos de Saúde Pública, Rio de Janeiro, v. 23, n. 2, p.399-407, 2007. 
CECCIM, Ricardo B.; FERLA, Alcindo A. Linha de cuidado: a imagem da mandala na gestão em rede de práticas cuidadoras para uma outra educação dos profissionais de saúde. In: PINHEIRO, Roseno; MATTOS, Ruben A. (Org). Gestão em redes: práticas de avaliação, formação e participação na saúde. Rio de Janeiro: CEPESC, 2006. p. 165-84.

FIGUEIREDO, Mariana D.; CAMPOS, Rosana O. Saúde mental e atenção básica à saúde: o apoio matricial na construção de uma rede multicêntrica. Saúde em Debate, Rio de Janeiro, v.32, n.78/79/80, p.143-9, 2008.

FRANCO, Túlio B.; MAGALHÃES J.R.; Helvécio M. Integralidade na Assistência à saúde: a organização das linhas do cuidado. In: MERHY, Emerson E. et al. (Org.). O Trabalho em Saúde: olhando e experienciando o SUS no cotidiano, o debate da saúde coletiva. São Paulo: Hucitec, 2006. p.125-33.

GONÇALVES, Alda M.; SENA, Roseni R. A Reforma psiquiátrica no Brasil: contextualização e reflexos sobre o cuidado com o doente mental na família. Revista Latino- Americana de Enfermagem, Ribeirão Preto, v. 9, n. 2, p. 48-55, 2001.

HARTZ, Zulmira M. A.; CONTADRIOPOULOS, André-Pierre. Integralidade da atenção e integração de serviços de saúde: desafios para avaliar a implantação de um "sistema sem muros". Cadernos de Saúde Pública, Rio de Janeiro, v. 20, supl. 2, p. 331-336, 2004.

IBGE. Instituto Brasileiro de Geografia e Estatística.Cidades@Rio Grande do Sul. Disponível em: <www.ibge.gov.br/cidadesat/ topwindow.htm? 1>. Acesso em: 2 mar. 2010.
LANCETTI, Antônio; AMARANTE, Paulo. Saúde Mental e Saúde Coletiva. In: CAMPOS, Gastão W. S; et al. (Org.). Tratado de Saúde Coletiva. São Paulo: Hucitec; Rio de Janeiro: Fiocruz, 2006. p. 615-33.

MINAYO, Maria C. S. (Org). O desafio do conhecimento: pesquisa qualitativa em saúde. São Paulo: Hucitec, 2007.

POLIT, Denise F.; BECK, Cheryl T.; HUNGLER, Bernadette P. Fundamentos da pesquisa em enfermagem: métodos, avaliação e utilização. Porto Alegre: Artmed, 2004.

SCHIMITH, Maria D.; LIMA, Maria A. D. S. Acolhimento e Vínculo em uma Equipe do Programa Saúde da Família. Cadernos de Saúde Pública, Rio de Janeiro, v. 20, n.6, p.1487-1494, 2004.

VASCONCELOS, Cipriano M.; PASCHE, Dário F. O Sistema Único de Saúde. In: CAMPOS, Gastão W.S.; (Org). Tratado de Saúde Coletiva. São Paulo: Hucitec; Rio de Janeiro: Fiocruz; 2006. p.531-62.

ZAMBENEDETTI, Gustavo; PERRONE, Claudia M. O processo de construção de uma rede de atenção em saúde mental: desafios e potencialidades no processo de reforma psiquiátrica. Physis - Revista de Saúde Coletiva, Rio de Janeiro, v. 18, n. 2, p. 277293, 2008.

Recebido em 25/05/2012

Aprovado em 22/08/2012 
